From the Departments of Radiology, Radiation Oncology, Neurology, and Biostatistics; and the Center for Molecular Imaging, University of Michigan Medical Center, Ann Arbor, Michigan.

Submitted November 6, 2007; accepted April 7, 2008; published online ahead of print at www.jco.org on June 9, 2008.

Supported by Grants No. P01CA85878, P01CA59827, 1P01CA87634 R24CA83099, and P50CA93990 from the National Institutes of Health and the National Cancer Institute.

Authors' disclosures of potential conflicts of interest and author contributions are found at the end of this article.

Corresponding author: Thomas L. Chenevert, PhD, University of Michigan, B2A209 UH 1500 East Medical Center Dr, Ann Arbor, Ml 48109-0030; e-mail: tlchenev@umich.edu.

(C) 2008 by American Society of Clinical Oncology

0732-183X/08/2620-3387/\$20.00 DOI: 10.1200/JCO.2007.15.2363

\title{
Functional Diffusion Map As an Early Imaging Biomarker for High-Grade Glioma: Correlation With Conventional Radiologic Response and Overall Survival
}

Daniel A. Hamstra, Craig J. Galbán, Charles R. Meyer, Timothy D. Johnson, Pia C. Sundgren, Christina Tsien, Theodore S. Lawrence, Larry Junck, David J. Ross, Alnawaz Rehemtulla, Brian D. Ross, and Thomas L. Chenevert

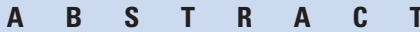

\section{Purpose}

Assessment of radiologic response (RR) for brain tumors utilizes the Macdonald criteria 8 to 10 weeks from the start of treatment. Diffusion magnetic resonance imaging (MRI) using a functional diffusion map (fDM) may provide an earlier measure to predict patient survival.

\section{Patients and Methods}

Sixty patients with high-grade glioma were enrolled onto a study of intratreatment $\mathrm{MRI}$ at 1,3 , and 10 weeks. Receiver operating characteristic curve analysis was used to evaluate imaging parameters as a function of patient survival at 1 year. Both log-rank and Cox proportional hazards models were utilized to assess overall survival.

\section{Results}

Greater increases in diffusion in response to therapy over time were observed in those patients alive at 1 year compared with those who died as a result of disease. The volume of tumor with increased diffusion by fDM at 3 weeks was the strongest predictor of patient survival at 1 year, with larger fDM predicting longer median survival (52.6 v 10.9 months; log-rank, $P<.003$; hazard ratio $[\mathrm{HR}]=2.7 ; 95 \%$ $\mathrm{Cl}, 1.5$ to 5.9). Radiologic response at 10 weeks had similar prognostic value (median survival, $31.6 \mathrm{~V}$ 10.9 months; log-rank $P<.0007 ; \mathrm{HR}=2.9 ; 95 \% \mathrm{Cl}, 1.7$ to 7.2). Radiologic response and fDM differed in $25 \%$ of cases. A composite index of response including fDM and RR provided a robust predictor of patient survival and may identify patients in whom RR does not correlate with clinical outcome.

\section{Conclusion}

Compared with conventional neuroimaging, fDM provided an earlier assessment of equal predictive value, and the combination of $\mathrm{fDM}$ and RR provided a more accurate prediction of patient survival than either metric alone.

\section{J Clin Oncol 26:3387-3394. (C) 2008 by American Society of Clinical Oncology}

\section{INTRODUCTION}

For malignant glioma, the Macdonald criteria are the primary radiologic response (RR) method, and have been correlated with survival. ${ }^{1-7}$ In addition, three-dimensional measurements of tumor volume have also been suggested to have a stronger association with survival. ${ }^{8}$ One disadvantage of size/volume measures is the time for changes to occur, ${ }^{1,9,10}$ with 8 to 10 weeks necessary to assess response.

Diffusion magnetic resonance imaging (MRI), which measures the random (Brownian) motion of water, has been proposed as an early biomarker for tumor response. ${ }^{11}$ Increased diffusion of water molecules (measured as an increase in the apparent diffusion coefficient [ADC]) occurs shortly after a successful treatment, and correlates with the break- down of cellular membranes and reduction in cell density that both precede changes in tumor size. Diffusion MRI has been evaluated in preclinical $^{12-27}$ and clinical studies. ${ }^{28-36}$ Quantification of diffusion changes has evolved from the mean change in $\mathrm{ADC}^{12,28}$ to a voxel-by-voxel approach termed the functional diffusion map (fDM). ${ }^{37-39}$ One potential disadvantage of the mean ADC is that different areas of tumor with increasing and decreasing changes in diffusion would cancel out, such that there would be no observed change in overall mean ADC, thus decreasing sensitivity. The $\mathrm{fDM}$, by measuring regional changes, is not limited in this manner and correlates with overall survival (OS) in a rodent glioma model. ${ }^{39}$ In patients with diverse primary brain tumors $^{38}$ or high-grade glioma, ${ }^{37}$ early changes in fDM (both increasing and decreasing diffusion) correlated 
with RR. In the present study, instead of correlating fDM with RR, itself a surrogate end point, we ascertained whether diffusion MRI could directly predict patient survival.

\section{PATIENTS AND METHODS}

\section{Patients}

Patients with primary brain tumors were enrolled onto a protocol of intratreatment MRI. We obtained informed consent, and the institutional review board approved images and medical record use. A total of 60 patients were evaluated on this study, of whom 34 were included in a previous analysis. ${ }^{37}$

\section{Treatment}

Radiotherapy was delivered using three-dimensional conformal therapy or intensity-modulated radiotherapy with at least 6-MV photons. Standard technique included a 2.0- to 2.5 -cm margin on either the enhancing region on gadolinium-enhanced scans or the abnormal signal on $\mathrm{T}_{2}$-weighted scans to 46 to $50 \mathrm{~Gy}$, with the gross tumor treated to a final median dose of $70 \mathrm{~Gy}$ in 6 to 7 weeks (Table 1 ). ${ }^{40}$ Twenty-one of these patients were treated on a phase 2 protocol of high-dose ( $>60 \mathrm{~Gy}$ ) radiation therapy concurrent with temozolomide. Chemotherapy was delivered as dependent on clinical circumstances (Table 1).

\section{Radiographic Scans}

Diffusion MRI and standard MRI (fluid attenuation inversion recovery, $\mathrm{T}_{2}$-weighted and gadolinium-enhanced $\mathrm{T}_{1}$-weighted $\mathrm{MRI}$ ) were performed 1 week before and 1,3 , and 10 weeks after the start of radiation with follow-up scans every 2 to 3 months.

\section{End Points}

$R R$ at 10 weeks was based on changes in tumor volume on $T_{1}$ contrastenhanced MRI and steroid doses and were classified as complete response (CR), partial response (PR), stable disease (SD), and progressive disease (PD). ${ }^{1}$ Steroid doses were recorded before each scan, weekly during radiotherapy, and at each follow-up.

\section{Diffusion MRI}

MRI scanning occurred on a General Electric (Waukesha, WI) 1.5T MRI system ( $\mathrm{n}=45$ patients) or a Philips (Best, the Netherlands) Achieva 3T system ( $\mathrm{n}=15$ patients). Diffusion imaging utilized a single-shot, spin-echo, diffusion-sensitized, echo-planar imaging (EPI) acquisition sequence. On the $1.5 \mathrm{~T}$ system, 246-mm axial-oblique sections were acquired using a $22 \mathrm{~cm}$-field of view $(F O V)$ and 128 matrix (voxel $=17.7 \mathrm{~mm}^{3}$ ) with "b-factor" $=0$ and 1,000 seconds $/ \mathrm{mm}^{2}$ along three orthogonal directions (repetition time $=$ $10,000 \mathrm{~ms}$; echo time $=71$ to $100 \mathrm{~ms}$, and number of averages $[\mathrm{NAV}]=1$ ). On the $3 \mathrm{~T}$ system, at least 28 4-mm axial-oblique sections were acquired through the brain using a 24-cm FOV and 128 matrix (voxel size $=14 \mathrm{~mm}^{3}$; repetition time $=2,636 \mathrm{~ms}$; echo time $=46 \mathrm{~ms} ; \mathrm{NAV}=1 \mathrm{for} \mathrm{b}=0$, and NAV $=2$ for $\mathrm{b}=$ 1,000 seconds $/ \mathrm{mm}^{2}$ ) with diffusion sensitization along three orthogonal directions. Parallel imaging (sensitivity encoding factor $=3$ ) was used at 3T to reduce spatial distortion. The diffusion images for the three orthogonal directions were combined to calculate an ADC map. ${ }^{41}$

\section{fDM Analysis}

Images were registered to the pretreatment MRI with a mutual information algorithmn, ${ }^{42}$ and regions of interest (ROIs) contoured on contrast-enhanced $\mathrm{T}_{1}$-weighted images. A minimum of $4 \mathrm{~mL}$ of tumor on postoperative scans was necessary for eligibility. If a resection cavity was present, it was included within the ROI if circumscribed by contrast enhancement and excluded if outside the enhancing region. Three patients did not have contrast-enhancing tumors before surgery, so ROI definition utilized all available MR sequences. Only voxels present in both the pretherapy and post-therapy tumor volumes were included for fDM analysis. Individual voxels were stratified into three categories based on the change in ADC from the pretreatment scan to each time point. Red voxels represented areas within the tumor where ADC increased $\left(>55 \times 10^{-5} \mathrm{~mm}^{2} /\right.$ $\mathrm{sec})$, blue voxels represented decreased ADC $\left(<55 \times 10^{-5} \mathrm{~mm}^{2} / \mathrm{sec}\right)$, and green voxels represented no change. This thresholds represent the $95 \% \mathrm{CI}$ for change (Fig 1) in ADC for uninvolved cerebral hemisphere. ${ }^{38}$ The percentage of the tumor within these three categories were calculated as $V_{I}$, $\mathrm{V}_{\mathrm{D}}$, and $\mathrm{V}_{0}$, respectively, and the total percentage of tumor with a significant change in diffusion values was $V_{T}$, where $V_{T}=V_{I}+V_{D}$.

\section{Statistical Analysis}

The thresholds for determining whether changes in volume, mean ADC, or fDM correlated with patient survival were determined using receiver operating characteristic (ROC) curve analysis. ROC curves identify the optimal threshold for a binary classifier using a graphical plot of sensitivity versus [1 1 -

Table 1. Receiver Operating Characteristic Curve Analysis of the Ability of Different Radiographic Criteria to Predict Patient Survival 1 Year From Diagnosis

\begin{tabular}{|c|c|c|c|c|c|c|c|c|}
\hline \multirow[b]{2}{*}{ Variable } & \multirow[b]{2}{*}{$A \cup C$} & \multicolumn{2}{|c|}{ Sensitivity } & \multicolumn{2}{|c|}{ Specificity } & \multirow[b]{2}{*}{ PPV } & \multirow[b]{2}{*}{ NPV } & \multirow[b]{2}{*}{$P$} \\
\hline & & $\%$ & $95 \% \mathrm{Cl}$ & $\%$ & $95 \% \mathrm{Cl}$ & & & \\
\hline \multicolumn{9}{|l|}{ Week 1} \\
\hline Change in volume, \% & 0.647 & 33.3 & 16.6 to 54.0 & 89.5 & 66.8 to 98.4 & 75.0 & 47.1 & $<.09$ \\
\hline Change in $A D C$ & 0.626 & 29.6 & 13.8 to 50.2 & 89.5 & 66.8 to 98.4 & 80.0 & 47.2 & $>.1$ \\
\hline fDM-V & 0.583 & 40.7 & 22.4 to 61.2 & 89.5 & 66.8 to 98.4 & 84.6 & 51.5 & $>.3$ \\
\hline \multicolumn{9}{|l|}{ Week 3} \\
\hline Change in volume, \% & 0.642 & 37.5 & 21.1 to 56.3 & 85.0 & 62.1 to 96.6 & 80.0 & 45.9 & $<.08$ \\
\hline Change in ADC & 0.687 & 53.1 & 34.8 to 70.9 & 85.0 & 62.1 to 96.6 & 85.0 & 53.1 & $<.02^{*}$ \\
\hline fDM-V & 0.758 & 68.8 & 50.0 to 83.9 & 85.0 & 62.1 to 96.6 & 88.0 & 63.0 & $<.0002^{*} \dagger$ \\
\hline \multicolumn{9}{|l|}{ Week 10} \\
\hline Change in volume, \% & 0.657 & 37.9 & 20.7 to 57.7 & 86.7 & 59.5 to 98.0 & 84.6 & 41.9 & $<.09$ \\
\hline Change in ADC & 0.632 & 29.6 & 13.8 to 50.2 & 86.7 & 59.5 to 98.0 & 80.0 & 40.6 & $>.1$ \\
\hline$f D M-V_{1}$ & 0.654 & 40.7 & 22.4 to 61.2 & 93.3 & 68.0 to 98.9 & 91.7 & 46.7 & $<.08$ \\
\hline Macdonald criteria & 0.671 & 65.6 & 46.8 to 81.4 & 70.0 & 45.7 to 88.0 & 77.8 & 56.0 & $<.04^{*} \dagger$ \\
\hline
\end{tabular}

NOTE. Volume and diffusion based parameters were evaluated 1, 3, and 10 weeks from the start of treatment. For sensitivity analysis the specificity for each test was set to approach $85 \%$ so that sensitivities could be compared across metrics for relatively similar specificities. Decreased ADC ( $<55 \times 10^{-5} \mathrm{~mm}^{2} / \mathrm{sec}$ and total percentage of tumor with a significant change in diffusion values were assessed at each time point but did not add predictive value and so are not presented.

Abbreviations: AUC, area under the curve; PPV, positive predictive value; NPV, negative predictive value; ADC, apparent diffusion coefficient; fDM, functional diffusion map; $V_{1}$, areas within the tumor where apparent diffusion coefficient increased $\left(>55 \times 10^{-5} \mathrm{~mm}^{2} / \mathrm{sec}\right)$.

*Initially statistically significant.

tStatistically significant variables after adjustment for multiple statistical comparisons. 

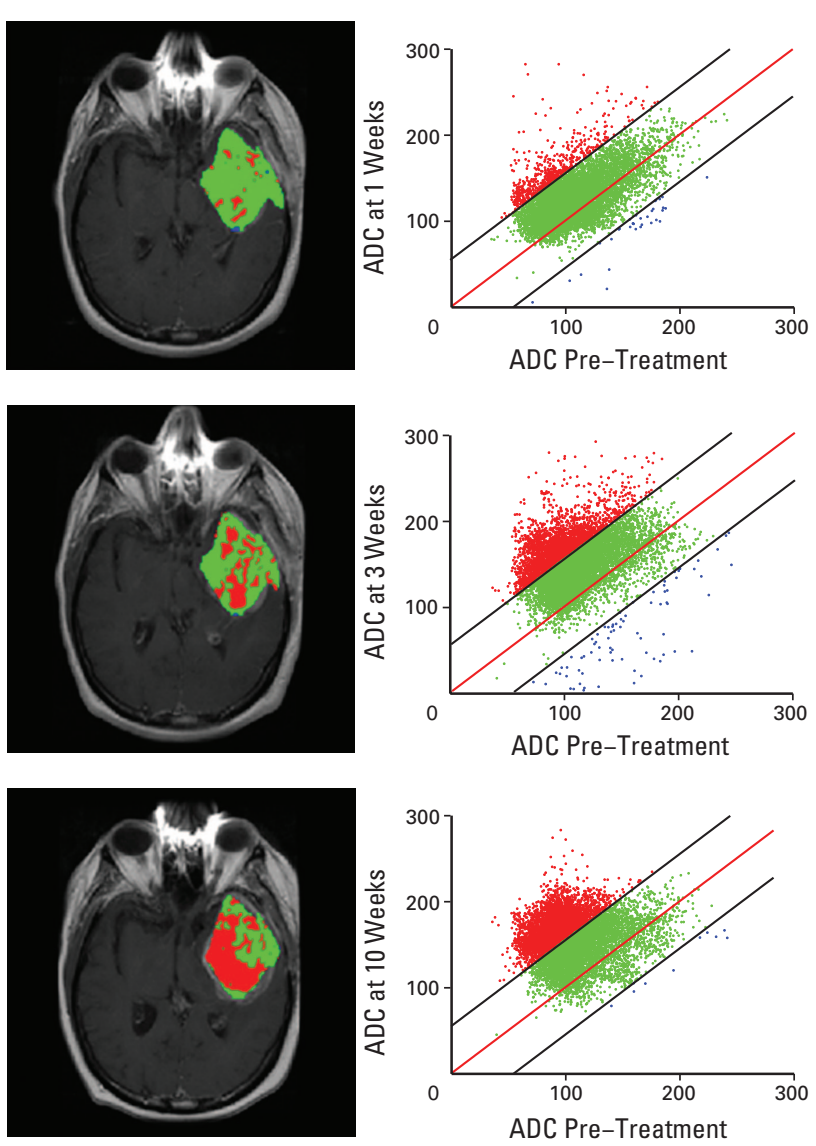
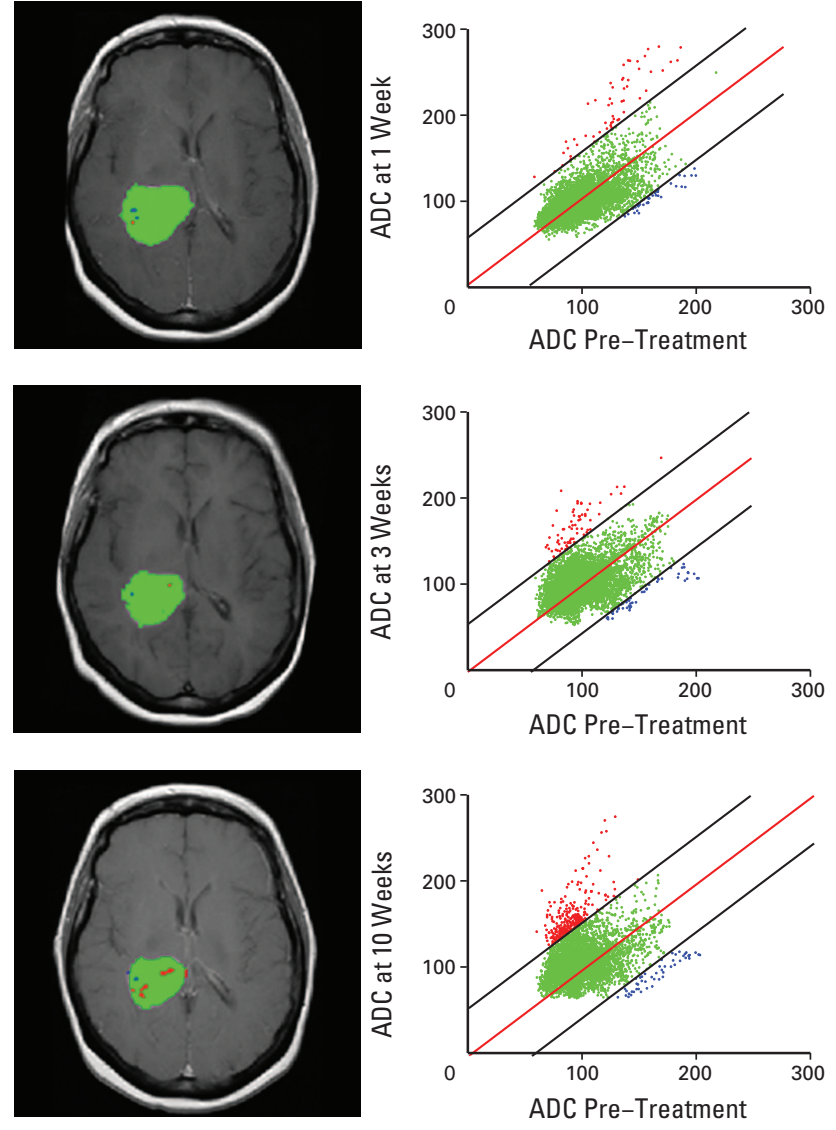

Fig 1. Representative functional diffusion map (fDM) analysis over time. Functional diffusion maps at (A) 1, (B) 3 , and (C) 10 weeks for two patients treated with

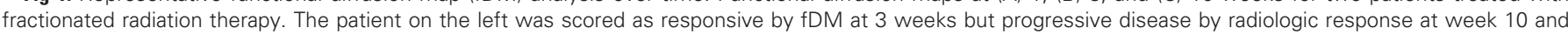

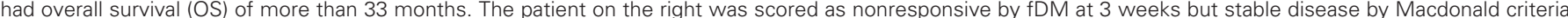

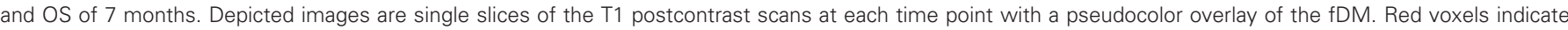

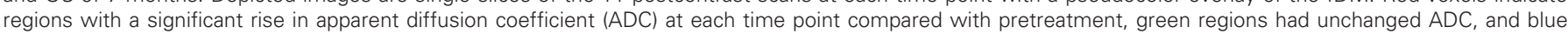

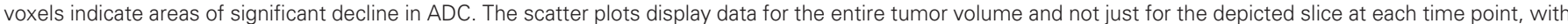

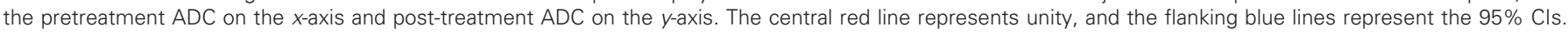

specificity]. The area under the curve (AUC) represents the overall predictive value across all thresholds, with perfect predictive value yielding $\mathrm{AUC}=1.0$. Optimizing a metric on a single data set introduces inherent bias (type III error) favoring a correlation with the end point of interest. Therefore, we performed leave-one-out cross-validation. This is a method for minimizing prediction error that involves leaving individual values out from the data set, performing the stratification, and then repeating the process " $n$ " number of times where $\mathrm{n}=$ the number of individual samples. This method results in an approximation of the unbiased estimate of the true predictive value. ${ }^{43}$ Given the 15 metrics evaluated by ROC curve analysis, a correction for multiple comparisons (Bonferroni correction) was applied such that only variables with an unadjusted $P$ of less than .0033 were considered significant. Differences based on categoric variables were assessed using Fisher's exact test, continuous variables utilized $t$ test, and trends were assessed with the Cochran-Armitage test. Survival analysis utilized log-rank and Cox proportional hazards models. Statistical analysis utilized MedCalc v9.3 (MedCalc Software, Mariakerke, Belgium).

\section{RESULTS}

\section{Patient Population}

Between November 1, 2000, and November 1, 2006, 70 patients with primary brain tumors were enrolled onto a prospective study of early tumor response. Sixty-seven of these patients had WHO grade 3 or IV astrocytoma, and 60 had assessable results form the population for this study. Seven patients were excluded for the following reasons: three had repeat surgical procedures within one month, one had claustrophobia, and three declined treatment. Pretreatment scans were performed 6 ( \pm 3.9 ) days from start of treatment, 54 patients had a scan at 1 week ( $6 \pm 2.8$ days), all 60 at 3 weeks ( $21 \pm 5.6$ days), and 55 at 10 weeks ( $71 \pm 14.2$ days). Median survival is 13.9 months, and at last contact, $30 \%$ of patients ( 18 of 60 ) were still alive with a median follow-up of 23.1 months.

\section{Evaluation of Response Measures}

A total of five metrics were measured at the three time points, including the percentage of change in tumor volume, the percentage of change in mean tumor $\mathrm{ADC}$, and three submetrics of $\mathrm{fDM}$ (increasing $\mathrm{ADC}\left[\mathrm{fDM}-\mathrm{V}_{\mathrm{I}}\right]$, decreasing $\mathrm{ADC}\left[\mathrm{fDM}-\mathrm{V}_{\mathrm{D}}\right]$, or any change in $\mathrm{ADC}\left[\mathrm{fDM}-\mathrm{V}_{\mathrm{T}}\right]$. Comparisons with standard $\mathrm{RR}$ were limited to the 55 patients with scans at 10 weeks. ROC curve analysis was performed to predict patient survival 1 year from diagnosis ( 34 of 55 
patients were alive 1 year from diagnosis, and 21 if 55 died; Table 2). All but two deaths were secondary to tumor progression.

\section{Change in Tumor Volume}

There were modest changes in tumor volume; median at 1, 3, and 10 weeks, respectively, was $+0.2 \%$ (interquartile range [IQR], -19.4 to +19.4 ), $+2.0 \%$ (IQR, -30.0 to 3.5 ), and $+0.3 \%$ (IQR, -32.2 to +56.6$)$. With smaller increases in volume at 10 weeks for those who were alive at 1 year compared with those who died (median, $-0.1 \%$ [IQR, -38.2 to +41.7$] v+46.9 \%$ [IQR, -17.5 to 122.2]; $P<.09$ ). By ROC curve analysis (Table 2) the change in tumor volume at each time point exhibited a trend toward predicting patient survival at 1 year but did not reach statistical significance $(P<.09$ at each time). When tumor response at 10 weeks was stratified by Macdonald criteria, this increased the predictive value (Table 2). No patient had CR, three had PR, 27 had SD, and 25 had $\mathrm{PD}$. The presence of SD or PR at 10 weeks was the best volumebased correlate with survival at 1 year $(P<.04)$.

\section{Changes in Mean ADC}

The changes in mean tumor ADC at 1,3 , and 10 weeks were, respectively, $+0.4 \%$ (IQR, -4.5 to +5.6 ), $+2.9 \%$ (IQR, -3.1 to
+6.9), and + 10.3\% (IQR, -1.6 to +21.6). Three-week mean ADC was associated with 1-year survival, with those alive exhibiting increased ADC (median, +3.4\% [IQR, -2.0 to + 12.0]) compared with a decreased ADC (median, $-1.5 \%$ [IQR, -6.9 to +0.9$]$ ) in those who died $(P<.03)$. By ROC curve analysis (Table 2$)$, the change in mean tumor ADC at 3 weeks was associated with 1-year survival $(P<.02)$ but the change at 1 and 10 weeks was not $(P>.1)$. After correcting for multiple comparisons, even the 3 -week metric was of only of borderline significance.

\section{Changes in fDM}

When regional tumor diffusion data were analyzed by fDM (Fig 1 ), the percentage of tumor with increasing diffusion $\left(\mathrm{fDM}-\mathrm{V}_{\mathrm{I}}\right)$ over time was associated with survival 1 year from diagnosis. $\mathrm{V}_{\mathrm{I}}$ increased linearly over time, with median increases of $1.6 \%$ (IQR, 0.4 to 4.3 ), $4.0 \%$ (IQR, 1.0 to 7.5 ), and $12.2 \%$ (IQR, 3.8 to 27.5 ) at 1,3 , and 10 weeks, respectively, with greater increases in $\mathrm{V}_{\mathrm{I}}$ for those alive at 1 year compared with those who had died (Cochran-Armitage $P<.001$; Appendix Fig A1, online only). To assess fDM as an early biomarker we focused on $V_{I}$ at 3 weeks. By ROC curve analysis the strongest relationship between any of the imaging metrics, and survival at 1 year

\begin{tabular}{|c|c|c|c|c|c|c|c|}
\hline \multirow[b]{3}{*}{ Variable } & & & \multicolumn{4}{|c|}{ fDM Category } & \multirow[b]{3}{*}{$P$} \\
\hline & \multicolumn{2}{|c|}{$\begin{array}{l}\text { All Patients } \\
(\mathrm{n}=60)\end{array}$} & \multicolumn{2}{|c|}{$\begin{array}{l}\text { Nonresponding } \\
\quad(n=29)\end{array}$} & \multicolumn{2}{|c|}{$\begin{array}{l}\text { Responding } \\
(\mathrm{n}=31)\end{array}$} & \\
\hline & No. & $\%$ & No. & $\%$ & No. & $\%$ & \\
\hline \multicolumn{8}{|l|}{ Pretreatment } \\
\hline Age, years & & & & & & & $<.03^{*}$ \\
\hline Median & \multicolumn{2}{|c|}{53} & \multicolumn{2}{|c|}{62} & \multicolumn{2}{|c|}{47} & \\
\hline Range & \multicolumn{2}{|c|}{$20-75$} & \multicolumn{2}{|c|}{$20-75$} & \multicolumn{2}{|c|}{$20-70$} & \\
\hline Pathology, grade & & & & & & & $>.5 \dagger$ \\
\hline 3 & 13 & 22 & 5 & 17 & 8 & 26 & \\
\hline 4 & 47 & 78 & 24 & 83 & 23 & 74 & \\
\hline KPS & & & & & & & $>.5 t$ \\
\hline$<90$ & 16 & 27 & 9 & 31 & 7 & 23 & \\
\hline$\geq 90$ & 44 & 73 & 20 & 69 & 24 & 77 & \\
\hline Location & & & & & & & $>.5 \dagger$ \\
\hline Frontal/temporal & 35 & 58 & 16 & 55 & 19 & 61 & \\
\hline Other & 25 & 42 & 13 & 45 & 12 & 39 & \\
\hline Mean tumor volume, $\mathrm{mL}$ & & & & & & & $>.5^{*}$ \\
\hline Median baseline ADC & & & & & & & $<.005^{*}$ \\
\hline \multicolumn{8}{|l|}{ Treatment related } \\
\hline Surgery & & & & & & & $<.002 \dagger \ddagger$ \\
\hline Biopsy & 26 & 43 & 19 & 66 & 7 & 23 & \\
\hline Subtotal & 18 & 30 & 5 & 17 & 13 & 32 & \\
\hline Near GTR & 16 & 27 & 5 & 17 & 11 & 45 & \\
\hline XRT dose, Gy & & & & & & & $>.3^{*}$ \\
\hline Mean & & & & & & & \\
\hline Range & & & & & & & \\
\hline \multicolumn{8}{|l|}{ Chemotherapy } \\
\hline Any & 56 & 93 & 27 & 93 & 29 & 94 & $1.0+$ \\
\hline Temozolomide & 54 & 90 & 26 & 90 & 28 & 90 & $1.0+$ \\
\hline Temozolomide + XRT & 26 & 43 & 11 & 38 & 15 & 48 & $>.4 t$ \\
\hline
\end{tabular}


was observed for $\mathrm{V}_{\mathrm{I}}$ at 3 weeks ( $P<.0002$; Table 2; Appendix Fig A2, online only).

Previously, ${ }^{37,38}$ both increasing and decreasing fDM at 3 weeks was correlated with RR at 10 weeks. In the present analysis, however, no correlation was found between patient survival at 1 year and decreasing diffusion by $\mathrm{fDM}(P>.1$ at 1,3 , and 10 weeks; Fig A1). Adding $V_{D}$ to $V_{I}$ (to yield $V_{T}$ ) was, therefore, associated with a lower predictive value for survival, and all analysis focused on $\mathrm{fDM}-\mathrm{V}_{\mathrm{I}}$ at 3 weeks.

\section{Optimization of fDM- $V_{1}$}

When assessed as a continuous variable, increasing $V_{I}$ at 3 weeks was correlated with increasing OS $(P<.02)$. Given the continuous nature of $\mathrm{V}_{\mathrm{I}}$, ROC curve analysis suggested a threshold of $4.7 \%$, where $V_{\text {I }} 4.7 \%$ or greater at 3 weeks was stratified as response and $\mathrm{V}_{\mathrm{I}}$ less than $4.7 \%$ as nonresponse. After leave-oneout cross-validation, $\mathrm{V}_{\mathrm{I}}$ remained a significant predictor of patient survival at 1 year $(P<.001$; AUC $=0.723$; sensitivity $=69.7 \%$ [95\% CI, 51.3 to 84.4 ]; specificity $=75.0 \%$ [ $95 \%$ CI, 50.9 to 91.2 ]; positive predictive value $[\mathrm{PPV}]=82.1 \%$; negative predictive value $[\mathrm{NPV}]=60.0 \%)$.

\section{Overall Survival As a Function of fDM Stratification and $R R$}

Using the $\mathrm{V}_{\mathrm{I}}$ threshold of $4.7 \%$, those with higher $\mathrm{V}_{\mathrm{I}}$ had median survival 52.6 months whereas those with lower $\mathrm{V}_{\mathrm{I}}$ had median survival of only 10.9 months $(P<.003 ; \mathrm{HR}=2.7 ; 95 \% \mathrm{CI}, 1.5$ to 5.9 ; Fig $2 \mathrm{~A})$. Conventional RR at 10 weeks was similarly prognostic (Fig 2B). Those with SD/PR had median survival of 31.6 months, whereas those with PD had median survival of 10.9 months $(P<.0007$; HR $=2.9 ; 95 \%$ CI, 1.7 to 7.2 ). For comparison with RR, fDM was limited to the 55 patients who had RR at 10 weeks, but if this analysis is extended to include all 60 patients, fDM was similarly prognostic $(P<.005$; $\mathrm{HR}=2.4 ; 95 \% \mathrm{CI}, 1.4$ to 4.8 ).

There was an association between RR and fDM stratification $(P<.001$; Fig 3$)$ with concurrence in $75 \%$ of cases ( 41 of 55$)$. Figure 1 presents two patients in whom fDM and RR differed in their stratification of response. The patient on the left was classified as PD by RR, but in contrast fDM documented a $\mathrm{V}_{\mathrm{I}}$ of $26.4 \%$ at 3 weeks (middle panel), and the patient was classified as responding by fDM. Despite $\mathrm{PD}$, this patient clinically stabilized and is alive without progression at 33 months. In contrast, the patient on the right had SD by RR, but had minimal change in tumor ADC at 3 weeks by fDM (middle panel, $1.6 \%$ ), clinically progressed within 5 months, and died at 7 months.

Given the differences between conventional RR and fDM in $25 \%$ of patients, a composite index of response was developed based on $\mathrm{FDM}$ and RR, and was the most robust response-based model for OS $(P<.0002$; Fig 2C). The composite identified three groups of patients. Those with the best prognosis were without radiographic progression (SD/PR) and responsive by $\mathrm{fDM}$, and had a median survival of 52.6 months. Those with the worst prognosis had low $\mathrm{V}_{\mathrm{I}}$ by fDM and PD by RR, and their median survival was 8.1 months. The intermediate group, comprising patients in whom fDM and RR differed, had a median survival of 14.4 months. Both the intermediate group $(P<.02 ; \mathrm{HR}=2.4 ; 95 \% \mathrm{CI}, 1.2$ to 4.6$)$ and the best-prognosis group $(P<.0001 ; \mathrm{HR}=4.2 ; 95 \% \mathrm{CI}, 2.4$ to 12.9$)$ were distinct from the worst-prognosis composite group.
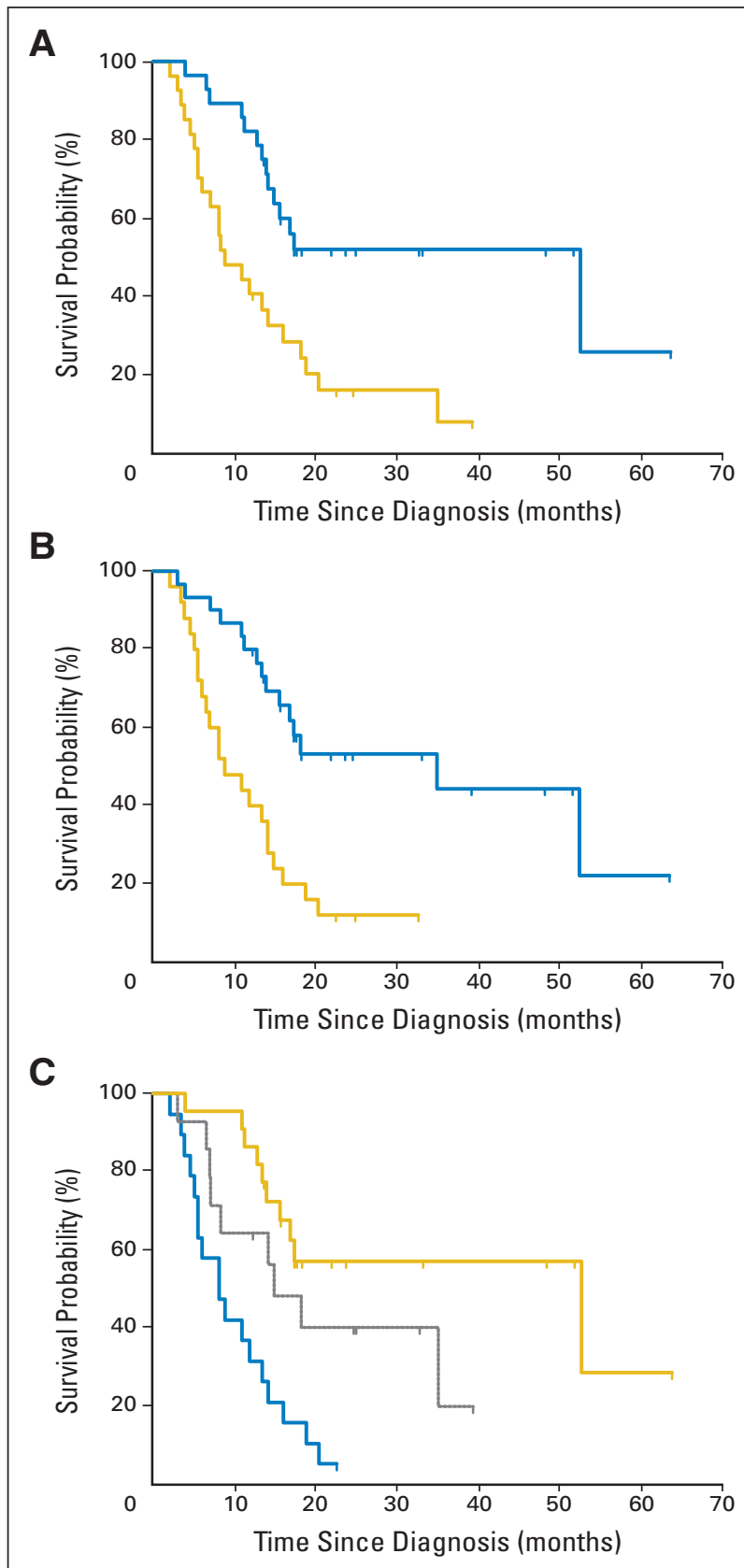

Fig 2. Overall survival as a function of functional diffusion map (fDM), radiologic response (RR), and their composite. (A) Overall survival by log-rank test based on fDM stratification at 3 weeks from the start of treatment where the yellow curve ( $n=27$ ) represents patients with $V_{1}<4.7 \%$ and the upper blue curve ( $n=28$ ) represents those with $V_{1} \geq 4.7 \%$. Median survival was 10.9 versus 52.6 months, respectively $(P<.003$; hazard ratio $[\mathrm{HR}]=2.7 ; 95 \% \mathrm{Cl}, 1.5$ to 5.9$)$. (B) Overall survival as a function of RR at 10 weeks from the start of treatment, where the yellow curve $(n=25)$ represents those patients with progressive disease and the upper blue curve represents those with stable disease $(n=27)$ or partial response $(n=3)$. Median survival was 10.9 versus 31.6 months, respectively $(P<.0007, \mathrm{HR}=2.9 ; 95 \% \mathrm{Cl}, 1.7$ to 7.2$)$. (C) Overall survival as a function of the composite index of response. The combination of fDM stratification at 3 weeks from the start of treatment and later radiographic response at 10 weeks provides a robust predictor of patient survival (log-rank $P<.0002$ ). Both the intermediate responding population (gray line, middle curve; $n=14$; median survival, 14.4 months; $P<.02$ ) and the best responding population (yellow line, top curve; $\mathrm{n}=$ 22; median survival, 52.6 months; $P<.0001)$ were distinct from the worstresponding radiographic group (blue line, bottom curve; $n=19$; median survival, 8.1 months). $V_{1}$, areas within the tumor where apparent diffusion coefficient increased ( $\left.>55 \times 10^{-5} \mathrm{~mm}^{2} / \mathrm{sec}\right)$. 


\section{Evaluation of Other Prognostic Variables}

To further assess the utility of fDM, we evaluated common variables previously found to correlate with survival in high-grade glioma (Table 1). Those disproportionately represented in the fDM responding group were younger age $(P<.03)$, higher baseline tumor ADC $(P<.005)$, and increased frequency of surgical resection $(P<.002)$. On univariate analysis, only age $(<50 v \geq 50$ years; $P<.006)$ and pathologic grade (WHO grade $3 v 4 ; P<.05$ ) correlated with OS. Performance status, surgical resection, pretreatment tumor ADC, use of chemotherapy, and radiation dose did not (Table 3). If limited to the patients with grade 4 tumors treated with definitive radiation therapy ( $\geq 60 \mathrm{~Gy} ; \mathrm{n}=41$ ) there was prolonged survival associated with concurrent temozolomide and radiation compared with radiation alone $(P<.05)$. When these individual variables were included in a multivariate model, only age and $\mathrm{fDM}$ were retained (Table 3 , model 1).

The best predictor of OS was the Radiation Therapy Oncology Group (RTOG) recursive partition analysis ${ }^{44}$ (RPA; Table 3; $P<.0004)$. When fDM was added to the RPA, both retained prognostic value (Table 3, model 2). Interestingly, across the five categories (there were no patients in class 2) there was an inverse relationship between class and the likelihood of response by fDM: $75 \%, 73 \%, 50 \%, 33 \%$, and $25 \%$, for classes $1,3,4,5$, and 6 , respectively $(P<.01$; Table A1, online only). For each class, median survival was longer in the group responding by fDM than in those not responding. Thus, although the numbers were small, it does appear that fDM retained prognostic value across the whole spectrum of disease. Patients predicted to have a worse outcome by RPA were also less likely to be responsive to therapy even as early as 3 weeks into treatment.

\section{DISCUSSION}

For glioma patients, the standard determination of RR is conventional MRI. ${ }^{1}$ In this study, RR based on the Macdonald criteria at 10 weeks did correlate with 1-year survival (PPV $=77.8 \%$ and $=$ NPV 56.0\%). Although this metric has been widely accepted, it does not allow for individualization of radiation treatment because the measurement is made well after the completion of therapy. Diffusion MRI evaluated using the fDM- $\mathrm{V}_{\mathrm{I}}$ at 3 weeks also correlated with patient survival at 1 year $(\mathrm{PPV}=82.1 \%$ and NPV $=60.0 \%)$, and might allow for response-based therapy alteration.

Interestingly, although $\mathrm{fDM}-\mathrm{V}_{\mathrm{I}}$ was prognostic at both 3 and 10 weeks, the greatest differentiation between responding and nonresponding tumors was observed at the early time point. It was previously noted that changes in diffusion MRI in both preclinical and clinical evaluations often precede volumetric response, and in fact, by the time tumors were documented to have responded by size criteria, many of the early changes observed by diffusion MRI had already resolved. ${ }^{12,28}$ Thus, although fDM was prognostic at both 3 and 10 weeks the greatest discrimination was observed before overt changes in tumor size had occurred, and fDM lost some prognostic value after early diffusion changes had dissipated.

The use of 3-week fDM- $\mathrm{V}_{\mathrm{I}}$ as an early biomarker for survival was at least as prognostic as the Macdonald criteria at 10 weeks, with similar PPV and NPV, but was obtained 7 to 8 weeks earlier. Combining $\mathrm{fDM}$ and RR into a composite provided the best response-based prediction, which provides an alternative use of fDM wherein current clinical care is maintained with the addition of fDM yielding a more accurate evaluation. This may help discern radiographic progression from "pseudoprogression," a recently identified clinical phenomenon wherein patients demonstrate radiographic evidence for progression of disease that may resolve without a change of treatment and without clinical progression. ${ }^{45}$ Size- and volume-based measures of response are also highly dependent on steroid dosing because these can influence tumor volume, blood vessel permeability, and contrast enhancement. In the current analysis, volume changes alone were significantly less prognostic than when steroid dosing was included in the evaluation, as in the Macdonald criteria. In contrast, diffusion changes within the gross tumor are largely unaffected after steroid treatment, whereas a moderate decline in peritumoral ADC has been observed after steroid treatment. ${ }^{46}$ Peritumoral edema was not included for fDM analysis and, therefore, steroid dosing did not influence fDM stratification.

At present, clinical variables are used to predict patient prognosis. However, there is also a growing body of genetic evidence that will certainly be used in the future to help identify the likelihood of a tumor's responding to treatment, such as specific genetic deletions, activation of oncogenes, loss of tumor suppressor genes, or promoter

\begin{tabular}{|c|c|c|c|c|c|}
\hline Variable & $\mathrm{HR}$ & $95 \% \mathrm{Cl}$ & Log Rank & Model 1 & Model 2 \\
\hline Age $(<50 v \geq 50$ years $)$ & 2.4 & 1.3 to 5.4 & $<.007$ & $<.03$ & \\
\hline Pathology (grade $3 \vee 4$ ) & 2.3 & 1.0 to 4.1 & $<.05$ & - & \\
\hline KPS $(<70 v \geq 70)$ & 1.1 & 0.5 to 3.0 & $>.5$ & - & \\
\hline Surgery (resection $v$ biopsy) & 1.6 & 0.8 to 3.2 & $>.1$ & - & \\
\hline RPA & NA & & $<.0004$ & - & $<.03$ \\
\hline$f D M-V_{1}(\geq 4.7 \% v<4.7 \%)$ & 2.7 & 1.5 to 5.9 & $<.003$ & $<.04$ & $<.04$ \\
\hline \multicolumn{6}{|c|}{$\begin{array}{l}\text { NOTE. Evaluated variables along with stratification thresholds for association with overall patient survival by single-variable (log rank) or multivariate (Cox proportional } \\
\text { hazards) models. HRs were obtained from log-rank evaluation. Model } 1 \text { evaluated individual pretreatment variables along with fDM; model } 2 \text { evaluated RPA stratification } \\
\text { and fDM. In each case, a step-wise analysis was used with variables at } P<.05 \text { retained in the final analysis. Bold text indicates statistical significance. } \\
\text { Abbreviations: HR, hazard ratio; KPS, Karnofsky performance score; ADC, apparent diffusion coefficient; XRT, radiotherapy; RPA, recursive partitioning analysis; NA, } \\
\text { not applicable; fDM, functional diffusion map; } V_{1} \text {, areas within the tumor where ADC increased }\left(>55 \times 10^{-5} \mathrm{~mm}^{2} / \mathrm{sec} \text { ). }\right.\end{array}$} \\
\hline
\end{tabular}




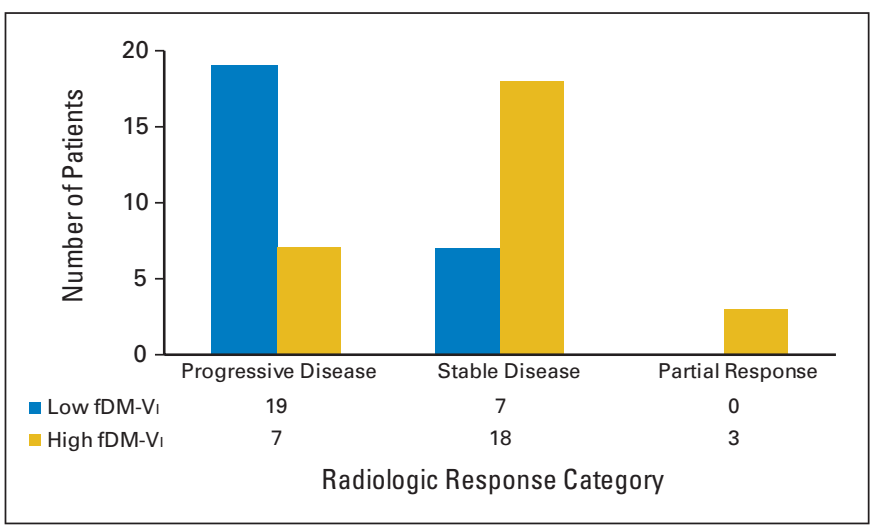

Fig 3. Correlation of functional diffusion map (fDM) mediated evaluation of response with radiologic response (RR). There was a strong correlation between increasing $f D M-V_{1}$ at 3 weeks and subsequent $R R$ at 10 weeks going from worst to best (progressive disease [PD] $\rightarrow$ stable disease [SD] $\rightarrow$ partial response; Cochran-Armitage $P<.001$ ). However, RR and fDM had conflicting results for $25 \%$ (14/55 patients) including seven patients with PD who demonstrated response by fDM and seven patients with SD who did not demonstrate response by fDM. $V_{1}$, areas within the tumor where apparent diffusion coefficient increased (>55 $\times 10^{-5} \mathrm{~mm}^{2} / \mathrm{sec}$ ).

methylation patterns. ${ }^{47-49}$ However, most of these tests have not been commonly adopted at present. A metric providing an early measure of actual tumor response - not just the likelihood of response-is critical and will have the capacity to add prognostic value across different genetic backgrounds.

Finally, the results reported in this article must be validated in a larger multi-institutional cohort before the fDM can be adopted as a biomarker for treatment response. In addition, although this study focused on glioma patients treated with radiation therapy with or without chemotherapy, the fDM can, in principle, be applied to most other cancers and treatments given that modern MRI scanners now allow diffusion measurements in other body regions with suitable motion compensation techniques. ${ }^{32-34,36,50}$
AUTHORS' DISCLOSURES OF POTENTIAL CONFLICTS OF INTEREST

Although all authors completed the disclosure declaration, the following author(s) indicated a financial or other interest that is relevant to the subject matter under consideration in this article. Certain relationships marked with a " $U$ " are those for which no compensation was received; those relationships marked with a " $C$ " were compensated. For a detailed description of the disclosure categories, or for more information about ASCO's conflict of interest policy, please refer to the Author Disclosure Declaration and the Disclosures of Potential Conflicts of Interest section in Information for Contributors.

Employment or Leadership Position: Alnawaz Rehemtulla, ImBio LLC (U); Brian D. Ross, ImBio LLC (U) Consultant or Advisory Role: None Stock Ownership: Alnawaz Rehemtulla, ImBIO LLC; Brian D. Ross, ImBio LLC Honoraria: Daniel A. Hamstra, Varian Medical Research Research Funding: None Expert Testimony: None Other Remuneration: None

\section{AUTHOR CONTRIBUTIONS}

Conception and design: Timothy D. Johnson, Alnawaz Rehemtulla, Brian D. Ross, Thomas L. Chenevert

Financial support: Brian D. Ross, Thomas L. Chenevert

Administrative support: Brian D. Ross

Provision of study materials or patients: Christina Tsien, Theodore S. Lawrence, Larry Junck

Collection and assembly of data: Thomas L. Chenevert

Data analysis and interpretation: Daniel A. Hamstra, Craig J. Galban, Charles R. Meyer, Timothy D. Johnson, Pia C. Sundgren, Christina Tsien, Theodore S. Lawrence, Larry Junck, David J. Ross, Alnawaz Rehemtulla, Brian D. Ross, Thomas L. Chenevert

Manuscript writing: Daniel A. Hamstra, Larry Junck, Brian D. Ross, Thomas L. Chenevert

Final approval of manuscript: Daniel A. Hamstra, Craig J. Galban, Charles R. Meyer, Timothy D. Johnson, Pia C. Sundgren, Christina Tsien, Theodore S. Lawrence, Larry Junck, David J. Ross, Alnawaz Rehemtulla, Brian D. Ross, Thomas L. Chenevert

\section{REFERENCES}

1. Macdonald DR, Cascino TL, Schold SC Jr, et al: Response criteria for phase II studies of supratentorial malignant glioma. J Clin Oncol 8:1277-1280, 1990

2. Barker FG II, Prados MD, Chang SM, et al: Radiation response and survival time in patients with glioblastoma multiforme. J Neurosurg 84:442-448, 1996

3. Gaspar LE, Fisher BJ, MacDonald DR, et al: Malignant glioma: Timing of response to radiation therapy. Int J Radiat Oncol Biol Phys 25:877-879, 1993

4. Wood JR, Green SB, Shapiro WR: The prognostic importance of tumor size in malignant gliomas: A computed tomographic scan study by the Brain Tumor Cooperative Group. J Clin Oncol 6:338-343, 1988

5. Lustig RA, Seiferheld W, Berkey $B$, et al: Imaging response in malignant glioma, RTOG 90-06. Am J Clin Oncol 30:32-37, 2007

6. Grant R, Walker M, Hadley D, et al: Imaging response to chemotherapy with RMP-7 and carboplatin in malignant glioma: Size matters but speed does not. J Neurooncol 57:241-245, 2002

7. Hess KR, Wong $E T$, Jaeckle $K A$, et al: Response and progression in recurrent malignant glioma. Neuro-oncol 1:282-288, 1999
8. Dempsey MF, Condon BR, Hadley DM: Measurement of tumor "size" in recurrent malignant glioma: 1D, 2D, or 3D? AJNR Am J Neuroradiol 26:770-776, 2005

9. Miller $A B$, Hoogstraten $B$, Staquet $M$, et al: Reporting results of cancer treatment. Cancer 47: 207-214, 1981

10. Therasse $P$, Arbuck SG, Eisenhauer EA, et al: New guidelines to evaluate the response to treatment in solid tumors. European Organization for Research and Treatment of Cancer, National Cancer Institute of the United States, National Cancer Institute of Canada. J Natl Cancer Inst 92:205-216, 2000

11. Hamstra DA, Rehemtulla A, Ross BD: Diffusion magnetic resonance imaging: $A$ biomarker for treatment response in oncology. J Clin Oncol 25: 4104-4109, 2007

12. Chenevert TL, McKeever PE, Ross BD: Monitoring early response of experimental brain tumors to therapy using diffusion magnetic resonance imaging. Clin Cancer Res 3:1457-1466, 1997

13. Chenevert TL, Ross BD: Diffusion, $T 1$, and $T 2$ as chemotherapeutic response predictors of experimental brain tumors. Proc Soc Magnet Resonance Med 2:924, 1995

14. Ross BD, Chenevert TL, Kim B, et al: Magnetic resonance imaging and spectroscopy: Applica- tion to experimental neuro-oncology. Q Magn Reson Biol Med 1:89-106, 1994

15. Ross BD, Moffat BA, Lawrence TS, et al: Evaluation of cancer therapy using diffusion magnetic resonance imaging. Mol Cancer Ther 2:581587, 2003

16. Schepkin VD, Chenevert TL, Kuszpit $K$, et al: Sodium and proton diffusion MRI as biomarkers for early therapeutic response in subcutaneous tumors. Magn Reson Imaging 24:273-278, 2006

17. Schepkin VD, Lee KC, Kuszpit K, et al: Proton and sodium MRI assessment of emerging tumor chemotherapeutic resistance. NMR Biomed 19: 1035-1042, 2006

18. Chinnaiyan AM, Prasad U, Shankar $S$, et al: Combined effect of tumor necrosis factor-related apoptosis-inducing ligand and ionizing radiation in breast cancer therapy. Proc Natl Acad Sci U S A 97:1754-1759, 2000

19. Galons JP, Altbach MI, Paine-Murrieta GD, et al: Early increases in breast tumor xenograft water mobility in response to paclitaxel therapy detected by non-invasive diffusion magnetic resonance imaging. Neoplasia 1:113-117, 1999

20. Poptani $\mathrm{H}$, Puumalainen $\mathrm{AM}$, Grohn $\mathrm{OH}$, et al: Monitoring thymidine kinase and ganciclovirinduced changes in rat malignant glioma in vivo by 
nuclear magnetic resonance imaging. Cancer Gene Ther 5:101-109, 1998

21. Stegman LD, Rehemtulla A, Hamstra DA, et al: Diffusion MRI detects early events in the response of a glioma model to the yeast cytosine deaminase gene therapy strategy. Gene Ther 7:1005-1010, 2000

22. Zhao M, Pipe JG, Bonnett J, et al: Early detection of treatment response by diffusionweighted $1 \mathrm{H}-\mathrm{NMR}$ spectroscopy in a murine tumour in vivo. Br J Cancer 73:61-64, 1996

23. Lee $K C$, Hall DE, Hoff BA, et al: Dynamic imaging of emerging resistance during cancer therapy. Cancer Res 66:4687-4692, 2006

24. Lee KC, Hamstra DA, Bhojani MS, et al: Noninvasive molecular imaging sheds light on the synergy between 5-fluorouracil and TRAIL/Apo2L for cancer therapy. Clin Cancer Res 13:1839-1846, 2007

25. Lee KC, Hamstra DA, Bullarayasamudram S, et al: Fusion of the HSV-1 tegument protein vp22 to cytosine deaminase confers enhanced bystander effect and increased therapeutic benefit. Gene Ther 13:127-137, 2006

26. Lee KC, Moffat BA, Schott AF, et al: Prospective early response imaging biomarker for neoadjuvant breast cancer chemotherapy. Clin Cancer Res 13:443-450, 2007

27. Lee KC, Sud S, Meyer CR, et al: An imaging biomarker of early treatment response in prostate cancer that has metastasized to the bone. Cancer Res 67:3524-3528, 2007

28. Chenevert TL, Stegman LD, Taylor JM, et al: Diffusion magnetic resonance imaging: An early surrogate marker of therapeutic efficacy in brain tumors. J Natl Cancer Inst 92:2029-2036, 2000

29. Mardor $Y$, Pfeffer $R$, Spiegelmann $R$, et al: Early detection of response to radiation therapy in patients with brain malignancies using conventiona and high b-value diffusion-weighted magnetic resonance imaging. J Clin Oncol 21:1094-1100, 2003

30. Roth $Y$, Tichler $T$, Kostenich G, et al: High-bvalue diffusion-weighted MR imaging for pretreatment prediction and early monitoring of tumor response to therapy in mice. Radiology 232:685692,2004
31. Hein PA, Kremser $C$, Judmaier $W$, et al: Diffusion-weighted magnetic resonance imaging for monitoring diffusion changes in rectal carcinoma during combined, preoperative chemoradiation: Preliminary results of a prospective study. Eur J Radiol 45:214-222, 2003

32. Kremser $C$, Judmaier $W$, Hein $P$, et al: Prelim inary results on the influence of chemoradiation on apparent diffusion coefficients of primary rectal carcinoma measured by magnetic resonance imaging. Strahlenther Onkol 179:641-649, 2003

33. Pickles MD, Gibbs $P$, Lowry $M$, et al: Diffusion changes precede size reduction in neoadjuvant treatment of breast cancer. Magn Reson Imaging 24:843-847, 2006

34. Hayashida Y, Yakushiij T, Awai K, et al: Monitoring therapeutic responses of primary bone tumors by diffusion-weighted image: Initial results. Eur Radiol 16:2637-2643, 2006

35. Mardor $Y$, Roth $Y$, Lidar $Z$, et al: Monitoring response to convection-enhanced taxol delivery in brain tumor patients using diffusion-weighted magnetic resonance imaging. Cancer Res 61:4971-4973, 2001

36. Kamel IR, Bluemke DA, Eng J, et al: The role of functional MR imaging in the assessment of tumor response after chemoembolization in patients with hepatocellular carcinoma. J Vasc Interv Radiol 17:505-512, 2006

37. Hamstra DA, Chenevert TL, Moffat BA, et al: Evaluation of the functional diffusion map as an early biomarker of time-to-progression and overall survival in high-grade glioma. Proc Natl Acad Sci U S A 102:16759-16764, 2005

38. Moffat BA, Chenevert TL, Lawrence TS, et al: Functional diffusion map: A noninvasive MRI biomarker for early stratification of clinical brain tumor response. Proc Natl Acad Sci U S A 102:5524-5529, 2005

39. Moffat BA, Chenevert TL, Meyer CR, et al: The functional diffusion map: An imaging biomarker for the early prediction of cancer treatment outcome. Neoplasia 8:259-267, 2006

40. Chan JL, Lee SW, Fraass BA, et al: Survival and failure patterns of high-grade gliomas after three-dimensional conformal radiotherapy. J Clin Oncol 20:1635-1642, 2002
41. Le Bihan D, Breton $E$, Lallemand $D$, et al: Separation of diffusion and perfusion in intravoxel incoherent motion MR imaging. Radiology 168:497505, 1988

42. Meyer CR, Boes JL, Kim B, et al: Demonstration of accuracy and clinical versatility of mutual information for automatic multimodality image fusion using affine and thin-plate spline warped geometric deformations. Med Image Anal 1:195-206, 1997

43. Hastie T, Tibshirani I, Friedman J: The Elements of Statistical Learning: Data Mining, Inference, and Prediction. New York, NY, Springer, 2001

44. Curran WJ Jr, Scott CB, Horton J, et al: Recursive partitioning analysis of prognostic factors in three Radiation Therapy Oncology Group malignant glioma trials. J Natl Cancer Inst 85:704-710, 1993

45. Taal W, Brandsma D, de Bruin HG, et al: The incidence of pseudo-progression in a cohort of malignant glioma patients treated with chemo-radiation with temozolomide. J Clin Oncol 25:77s, 2007 (suppl; abstr 2009)

46. Armitage PA, Schwindack C, Bastin ME, et al: Quantitative assessment of intracranial tumor response to dexamethasone using diffusion, perfusion and permeability magnetic resonance imaging Magn Reson Imaging 25:303-310, 2007

47. Hegi ME, Diserens AC, Gorlia T, et al: MGMT gene silencing and benefit from temozolomide in glioblastoma. N Engl J Med 352:997-1003, 2005

48. Cairncross JG, Ueki K, Zlatescu MC, et al: Specific genetic predictors of chemotherapeutic response and survival in patients with anaplastic oligodendrogliomas. J Natl Cancer Inst 90:1473-1479, 1998

49. Mellinghoff IK, Wang MY, Vivanco I, et al: Molecular determinants of the response of glioblastomas to EGFR kinase inhibitors. N Engl J Med 353:2012-2024, 2005

50. Lee KC, Bradley DA, Hussain M, et al: A feasibility study evaluating the functional diffusion map as a predictive imaging biomarker for detection of treatment response in a patient with metastatic prostate cancer to the bone. Neoplasia 9:1003-1011, 2007

\section{Appendix}

The Appendix is included in the full-text version of this article, available online at www.jco.org. It is not included in the PDF version (via Adobe ${ }^{\circledR}$ Reader $^{\circledR}$ ). 